\title{
Fachkunde des Datenschutzbeauftragten
}

\author{
Johann Bizer
}

Voraussetzung der Bestellung eines betrieblichen Datenschutzbeauftragten durch die verantwortliche Stelle ist, dass er die ,zur Erfüllung seiner Aufgaben erforderliche Fachkunde" besitzt ( $\$ 4 \mathrm{f}$ Abs. 2 Satz 1 BDSG). Ist der Beauftragte bestellt, dann ist er ,,in Ausübung seiner Fachkunde auf dem Gebiet des Datenschutzes" weisungsfrei ( $§$ 4 f Abs. 3 Satz 2 BDSG). Seine Qualifizierung muss ihn also befähigen, fachlich eigenverantwortliche Entscheidungen $\mathrm{zu}$ fällen. Die Bedeutung der Fachkunde des Beauftragten wird dadurch unterstrichen, dass er von der Aufsichtsbehörde abberufen werden kann, ,wenn er die zur Erfüllung seiner Aufgaben erforderliche Fachkunde" nicht besitzt ( $\$ 38$ Abs. 5 Satz 3 BDSG).

Welche Fachkunde als Voraussetzung seiner Bestellung erforderlich ist, ist funktional aus seinen gesetzlichen Aufgaben zu bestimmen.

\section{Compliance}

Aufgabe des Beauftragten ist es, auf die „Einhaltung der Gesetze und anderer Vorschriften über den Datenschutz" hinzuwirken“ (§ 4 g Abs. 1 Satz 1 BDSG). Gemeint sind sowohl die staatlichen Regelungen als auch die unternehmensinternen Regelwerke von Betriebsvereinbarungen bis hin zu internen Dienstanweisungen.

Compliance ist mit „Normbefolgung“ zu übersetzen. Die Regeln des Datenschutzes sind nur ein Ausschnitt einer umfassenderen Organisationsaufgabe im Unternehmen. Es muss staatliche Regelwerke einhalten, was angesichts der Vielzahl an Regelungen vor allem auch für Unternehmen mit Aktivitäten in unterschiedlichen Ländern eine Herausforderung darstellt.

Compliance hat aber auch nach Innen eine Bedeutung: Die Unternehmensleitung kann das Unternehmen nur dann verantwortlich steuern, wenn ihre Direktiven auch von den untergeordneten Einheiten befolgt werden. Der Datenschutzbeauftragte leistet mit anderen Worten seinen Beitrag zur Unternehmenskultur der Normbefolgung, indem er auf die Einhaltung datenschutzspezifischer Regeln und Anweisungen hinwirkt.
Fachkunde zur Datenschutz-Compliance bedeutet nicht nur Kenntnis der einschlägigen Datenschutzbestimmungen, sondern auch der unternehmensinternen Mechanismen, ihre Befolgung zu überprüfen sowie ihrer Umsetzung durchzusetzen.

\section{Riskmanagement}

Die Fachkunde muss den Beauftragten auch dazu befähigen, seine Aufgabe im Rahmen des Riskmanagment im Unternehmen wahrzunehmen. Das BDSG formuliert in dieser Hinsicht zwei zentrale Aufgaben des Beauftragten: Zum einen die Überwachung der „Ordnungsmäßigkeit der Datenverarbeitung“ (§ 4 g Abs. 1 Satz 3 Nr. 1 BDSG) sowie die Prüfung und Bewertung der automatisierten Datenverarbeitungen im Rahmen der Vorabkontrolle ( $\$ 4 \mathrm{~d}$ Abs. 5 BDSG).

Beide Aufgaben beschränken sich nicht auf die Kontrolle, ob Anforderungen erfüllt sind, sondern im Interesse eines proaktiven Riskmanagements gilt das Augenmerk der Vorsorge im Wege einer Beratung der Unternehmenseinheiten. Dies setzt Kenntnisse der datenschutzrechtlichen Anforderungen sowie ihrer Umsetzung in technisch-organisatorischen Maßnahmen voraus. Sie erfordern darüber hinaus eine anwendungsbezogene Schutzbedarfsanalyse sowie eine Risikoabschätzung.

Der Beauftragte muss also über Kenntnisse und Erfahrungen des unternehmensinternen Riskmanagements in der Informationstechnik verfügen. Er muss nicht nur Wissen, „welche" Anforderungen umzusetzen sind, sondern auch auf ,welche Weise“. Die Einbettung des Monitoring in die jeweilige unternehmensspezifische Unternehmenskultur wird entscheidend für ein erfolgreiches Riskmanagement durch den Beauftragten sein

\section{Awareness}

Eine weitere Aufgabe des Beauftragten, ist die Mitarbeiterschulung ( $\$ 4$ g Abs. 1 Satz 3 Nr. 2 BDSG). Sie ist Bestandteil des Riskmanagements. Die Mitarbeiter sollen mit Ziel und Inhalt der Datenschutzvorschriften vertraut gemacht werden, damit durch diese
Sensibilisierung Schäden vermieden werden können. Awareness setzt Vermittlungskompetenzen voraus. Je besser die Qualifizierung der Mitarbeiter, desto geringer der spätere Kontrollaufwand.

\section{Kommunikative Kompetenz}

Fachkunde ist mehr als nur Wissen und Erfahrung - sie erfordert vor allem auch eine kommunikative Kompetenz, Anforderungen $\mathrm{zu}$ analysieren und Lösungsmodelle im Wege der Beratung zu vermitteln.

Das BDSG verdeutlicht diese Anforderungen an drei Stellen: Der Beauftragte ist unmittelbar der Leitung der verantwortlichen Stelle unterstellt ( $\$ 4 \mathrm{f}$ Abs. 3 Satz 1 BDSG). Diese Stellung erfordert von dem Beauftragten, Fingerspitzengefühl und argumentative Überzeugungskraft, um die Anforderungen des Datenschutzes und seiner Bedeutung gegenüber der Leitung Geltung verschaffen zu können.

Auch die Weisungsfreiheit des Beauftragten in Ausübung seiner Fachkunde $(\S 4 \mathrm{f}$ Abs. 3 Satz 2 BDSG) ist ohne kommunikative Kompetenz nicht auszufüllen. Wer sich auf seine Weisungsfreiheit erst berufen muss, hat erfahrungsgemäß einen schlechten Stand. Umgekehrt gilt, dass die Weisungsfreiheit nicht in Anspruch genommen werden muss, wenn die fachliche Kompetenz des Beauftragten evident ist. Sie wird also gelebt und nicht bloß behauptet.

Eine weitere kommunikative Kompetenz ergibt sich aus der Verpflichtung des Beauftragten zur Verschwiegenheit ( $\$ 4 \mathrm{f}$ Abs. 4 BDSG). Schweigen zu können, ist eine Tugend. Sie erfordert persönliche Stabilität und Lebenserfahrung, insbesondere in emotionalen Konfliktlagen.

Fazit

Die Anforderungen an die Fachkunde sind in Hinblick auf die Managementziele des Compliance und des Riskmanagement auszulegen. Dies erfordert von dem betrieblichen Datenschutzbeauftragten Kenntnisse und Erfahrungen in den Bereichen Recht, Technik, Management sowie Kommunikative Kompetenz. 